\title{
Non-Linear Effects in KNOWLedge PRODUCTION
}

\author{
lonuț PURICA*
}

\begin{abstract}
The generation of technological knowledge is paramount to our present development. Economic science concentrates on representing the functions of production applied to all sectors, e.g., the well known Cobb-Douglas model, associated with parameters such as capital and labor. Based on the paradigm, demonstrated in another paper, that the production of technological knowledge is governed by the same Cobb-Douglas type model, by the means of research and the intelligence level replacing capital, respectively labor, we are exploring the basic behavior of present days economies that are producing technological knowledge, along with the 'usual' industrial production. Considering the intercorrelations of technology and industrial production we determine a basic behavior that turns out to be a 'Henon attractor', well known as one of the first analyzed systems that present chaotic behavior confined to strange attractors. The behavior inside the basin of the attractor's dynamic shows some interesting features such as the fact that too little effort in technological knowledge production is associated to low industrial production, while too much resource allocation to technological production is also reaching an area of low industrial production. This effect clearly shows that too little allocation of resources to research is equivalent to a disproportionate allocation of resources to research, namely that both hamper the industrial production. Moreover, there is an area of large industrial production that corresponds to a certain rate of technology production that, in some way, optimizes development. Measures are introduced for the gain of technological knowledge and the information of technological sequences that are based on the underlying multi-valued logic of the technological research and on nonlinear thermodynamic considerations. We have witnessed in the last decades several cases of economies, e.g., Ireland and Finland, in Europe, the Asian tigers and China in Asia, which had had a moment in their history when research (both means and intelligence) was a main priority. Luckily, the globalization acted as a stabilizer that kept them close to the optimum of 'As High As Reasonably Acceptable' technological production. By contrast to ALARA (as low as reasonably acceptable) principle, that applies in risk analysis, here we may introduce the AHARA principle resulting from the nonlinear behavior of technological production vs. industrial production.
\end{abstract}

Key Words: knowledge management, strange attractors, experimental state of knowledge

JEL Classification: O14, O3, C62, C80, C99, D8.

* Institute of Economic Forecasting - Romanian Academy, puricai@yahoo.com. 


\section{Non-Linear Effects in Knowledge Production}

\section{Nonlinear effects in the economic systems}

Let us analyze the way "hill-shaped" dynamic relationships can arise in economics starting with a simple example showing this pattern:

Consider the relationship between a firm's profits and its advertising budget decision. Suppose that without any expenditure on advertising the firm cannot sell anything. As advertising outlay rises, total net profit first increases, then gradually levels off and finally begins to decline, yielding the traditional hill-shaped profit curve. If $P_{t}$ represents total profit in period $t$ and $y_{t}$ is total advertising outlay, $P_{t}$ can, for illustration, be expressed as $\mathrm{P}_{\mathrm{t}}=\mathrm{ay}_{\mathrm{t}}\left(\mathrm{l}-\mathrm{y}_{\mathrm{t}}\right)$. If the firm devotes a fixed proportion, $\mathrm{b}$, of its current profit to advertising outlays in the following period so that $\mathrm{y}_{\mathrm{t}+1}=\mathrm{bP} \mathrm{P}_{\mathrm{t}}$, the first equation is transformed into our basic chaos one with $w=a b$.

The reason the slope of the phase graph turns from positive to negative in this case is clear and widely recognized. Even if an increase in advertising outlay always raises total revenue, after a point its marginal net profit yield becomes negative and, hence, the phase diagram exhibits a hill shaped curve.

Giving it some thought one may see why the time path of $y_{t}$ can be expected to be oscillatory. Suppose the initial level of advertising, $y_{0}$, is an intermediate one that yields a high profit figure $P_{0}$. That will lead to a large (excessive) advertising outlay $y_{1}$ in the next period, thereby bringing down the value of profit figure $P_{1}$. That, in turn, will reduce advertising again and raise profit and so on ad infinitum.

The thing to be noted about this process is that it gives good reason to expect the time paths of profit and advertising expenditures to be oscillatory. But it does not give us any reason to expect that these time paths need either be convergent or perfectly replicatory. This is an example of how chaotic behavior patterns can be.

Another example has been provided in the theory of productivity growth (Baumol and Wolff, 1983). It involves the relationship between the rate of productivity growth $\left(P_{t}\right)$ and the level of R\&D expenditures by private industry ( $r$ ). Obviously a rise in $r$ can be expected to increase $P_{t}$. However since research can be interpreted as a service activity with a more or less fixed labor component, its costs will be raised by productivity growth in the reminder of the economy and the resulting stimulus to real wages. This, in turn, will cut back the quantity of R\&D demanded. The result, as a formal model easily confirms, will be a cycle with high productivity rates leading to high R\&D prices which restrict the next period's productivity growth, and so reduce R\&D prices, and so on. If R\&D costs ultimately increase disproportionately with increases in productivity growth it is clear that the relation $P_{t+1}=f\left(P_{t}\right)$ can generate the sort of hill-shaped phase graph that is consistent with a chaotic regime.

Another model that can generate cyclical or chaotic dynamics is a standard growth model of Solow type in which the propensity to save out of wages is lower than that for profits. Suppose that at low levels of capital stock $\mathrm{K}$ one obtains increasing marginal returns to increased capital and the elasticity of substitution of labor for capital is initially low; but diminishing returns eventually set in and the elasticity of substitution moves the other way. Then total profits can rise, at first, relative to total wages, but later profits may fall both relative to wages, and even absolutely. This can generate a 
hill-shaped relationship between $\mathrm{K}_{\mathrm{t}+1}$ and $\mathrm{K}_{\mathrm{t}}$ as rising $\mathrm{K}_{\mathrm{t}}$ at first elicits rising savings and eventually depresses them as profits fall.

\section{Earlier dynamic models}

The roots of the economists' interest in complex dynamics are to be found in the nonmathematical literature on business cycles, with its large number of models, undertaking to provide a set of conditions sufficient to generate oscillatory behavior in economy.

In the 1930's the work of Frisch (1933), Lundberg (1937) and Samuelson (1939) started using difference and differential equations in models that generated deterministic time paths.

A non trivial example is Samuelson's multiplier accelerator model (1939) which is made up of the three standard relationships:

$$
\mathrm{Y}_{\mathrm{t}}=\mathrm{C}_{\mathrm{t}}+\mathrm{I}_{\mathrm{t}} \quad \mathrm{C}_{\mathrm{t}}=\mathrm{cY}_{\mathrm{t}}+\mathrm{k} \quad \mathrm{I}_{\mathrm{t}}=\mathrm{b}\left(\mathrm{Y}_{\mathrm{t}-1}-\mathrm{Y}_{\mathrm{t}-2}\right)
$$

where $\mathrm{Y}$ is national income (output), $\mathrm{C}$ is consumption, $\mathrm{c}$ is the marginal propensity, and $\mathrm{I}$ is investment. The $\mathrm{C}$ equation is a linear consumption production with a oneperiod lag. The investment function is a linear lagged accelerator with investment assumed proportionate to the preceding period's rate of output growth. Substitution of the latter two equations in the last one yields

$$
\mathrm{Y}_{\mathrm{t}}=(\mathrm{C}+\mathrm{b}) \mathrm{Y}_{\mathrm{t}-1}-\mathrm{bY}_{\mathrm{t}-2}+\mathrm{k}
$$

which is Samuelson's second order linear difference equation.

Out of these types of model four qualitative behaviors were generated:

1) oscillatory and stable (i.e. converging with oscillations of decreasing amplitude toward some fixed equilibrium value);

2) oscillatory and explosive (cycles with divergent amplitude);

3) non-oscillatory and stable;

4) non-oscillatory and explosive.

It was soon recognized that linear equations of an even higher order than Samuelson's would not generate time paths any different from the ones above. This range of possible time paths configurations simply was not sufficiently rich for the economists' purposes, since in reality time paths are often more complex and many oscillations do not seem to explode or dampen toward disappearance.

A solution, brought to attention by Hicks and Goodwin, was the nonlinear modeling, considered of a general form:

$$
y_{t}=f\left(y_{t-1}, \ldots, Y_{t-h}\right)
$$

Responding to real economic issues these authors, for example, showed that such a non-linear model can yield a stable limit cycle toward which all possible time paths of the variable $Y_{t}$ converge. Matters were left here, the work stopping short of introducing a degree of non-linearity sufficiently great to generate chaotic behavior. 


\section{Non-Linear Effects in Knowledge Production}

\section{Essentials of the chaotic behavior}

In essence, chaos theory shows that a simple relationship that is deterministic but non-linear, such as a first order nonlinear difference equation, can yield an extremely complex time path.

Inter-temporal behavior can acquire an appearance of disturbance by random shocks and can undergo violent, abrupt qualitative changes, either with the passage of time or with small changes in the values of the parameters. Chaotic time paths can have the following attributes, among others:

a) a trajectory (time path) can sometimes display sharp qualitative changes in behavior, like those we associate with large random disturbances (e.g. very sudden changes from small amplitude to large amplitude cycles and vice versa), so at least some tests of randomness cannot distinguish such chaotic patterns of change from "truly random" behavior;

b) a time path is sometimes extremely sensitive to microscopic changes in the values of the parameters which can completely transform the qualitative character of the path;

c) they may display in a bounded region an oscillatory pattern which is very "disorderly".

Chaos theory provides, both for the economic analyst and for the policy designer, warning signals that apparently random behavior may not be random at all, demonstrating the dangers of extrapolation and showing the difficulties that can beset economic forecasting generally.

\section{Complex cyclical patterns}

The simplest and most common chaos model involves a nonlinear one-variable difference equation of first order, that is, one of the form:

$$
y_{t+1}=f\left(y_{t}\right)
$$

whose graph (the phase diagram) showing $f\left(y_{t}\right)$ as a function of $y_{t}$ is hill-shaped and tunable; in other words, the height, steepness, and location of the hill can be adjusted as desired by a suitable modification in the values of the parameters of $f\left(y_{t}\right)$, This phase diagram is the geometric instrument used to analyze the chaotic behavior time path generated by a difference equation model.

The function most commonly used to illustrate the chaos phenomenon is the quadratic equation with a single parameter, $w$ :

As it may be seen from the equation, if

$$
y_{t+1}=f\left(y_{t}\right)=w y_{t}\left(1-y_{t}\right) ; \text { where } d y_{t+1} / d y_{t}=w\left(1-2 y_{t}\right)
$$

$w<1$ the phase curve will lay bellow the $45^{\circ}$ line in the first quadrant;

$w>1$ there will be a positive value equilibrium point at the intersection of the $45^{\circ}$ line and the parabolic curve;

$1<w<2$ the phase curve slope at the intersection point will be positive;

$2<w<3$ the slope will be negative but less than unity in absolute value;

$w>3$ the slope will be less than -1 . 
The last case is the one when the chaotic behavior may set in after a number of frequency bifurcation of the emerging limit cycles (from 2 frequencies to 4 frequencies, etc.). Since, for example, Grandmond and Malgrange (1986) and Baumol and Benhabib (1989) give extensive descriptions of the phase space of this equation for the economic dynamic case.

\section{The industrial production and the production of technologies}

We will not insist here on the demonstration of the fact that the production of technological knowledge is behaving similar to the industrial production modeled by Cobb-Douglas formulae with different meaning of the parameters and variables. Purica, 1988, gives an in-depth analysis and his results will be taken here and used for developing a simple model that is beyond a separate analysis of the two types of production in trying to assess their mutual correlations.

First, to visualize the correlation of the two types of production, the figure below (Purica, 1988) gives the correlation diagram of the industrial production and the production of technologies. The symmetric relations of the two are straight forward.

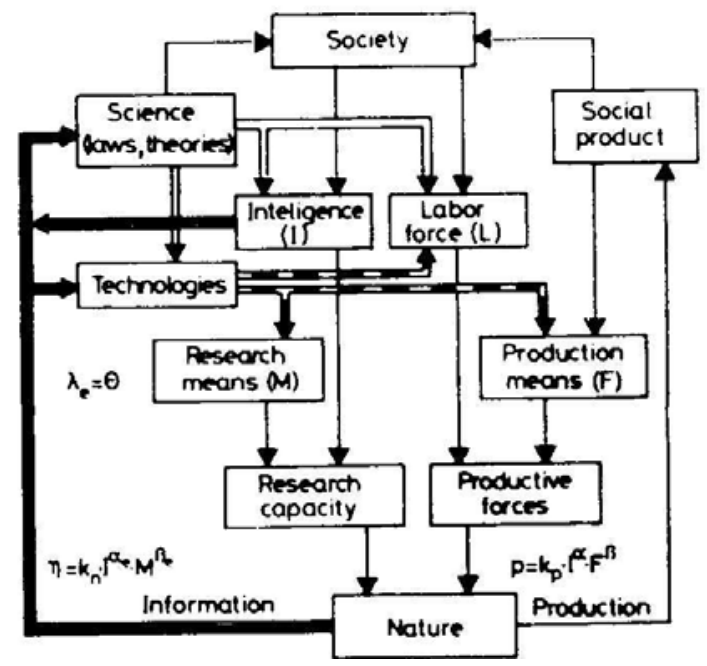

Having presented the similar behavior of the industrial production and the production of technological knowledge we will consider in what follows how the two are correlated.

A simple consideration would state that the production of technologies is proportional to the allocated part, of the previous year industrial production, to technological research. Denoting by $t(n)$ the production of technologies of the current year and by $p(n-1)$ the industrial production of the previous year, we may write:

$$
t(n)=b^{*} p(n-1)
$$




\section{Non-Linear Effects in Knowledge Production}

On the other side, we assess that the industrial production of this year will depend on the result of technologies production of the previous year, $t(n-1)$, and also on a more complex term reflecting the combined influence of the previous year production and of the technologies of this year. Thus we may write, with the above notation:

$$
p(n)=1+t(n-1)+a^{\prime *} p(n-1)^{*} t(n)
$$

and, considering the relation for $t(n)$, the equation for $p(n)$ becomes:

$$
p(n)=1+t(n-1)+a^{*} p(n-1)^{*} p(n-1)
$$

In the formula above we introduced a constant, scaled to 1 , that is bringing the expression of $p$ closer to the usual Cobb-Douglas expressed as a sum of logarithms. For example we may consider that the disappearance of the unqualified labor (periodic training of labor to cope with higher productivity brought by new technologies) is showing that a measure of labor is given by the amount of technological knowledge produced, while the capital becomes more efficient, i.e. square of previous production, due to the implementation of new technologies. These last statements are just conjectures that need to be proved by experimental analysis on existing data for various economies. Just for reference, the data on industrial production and patents for the USA starting from 1860 till 1976, given by Haustein and Neurath in 1982, are showing, as analyzed here, a dependence that is non-linear (see figure below).

\section{Patents vs. industrial production in USA}

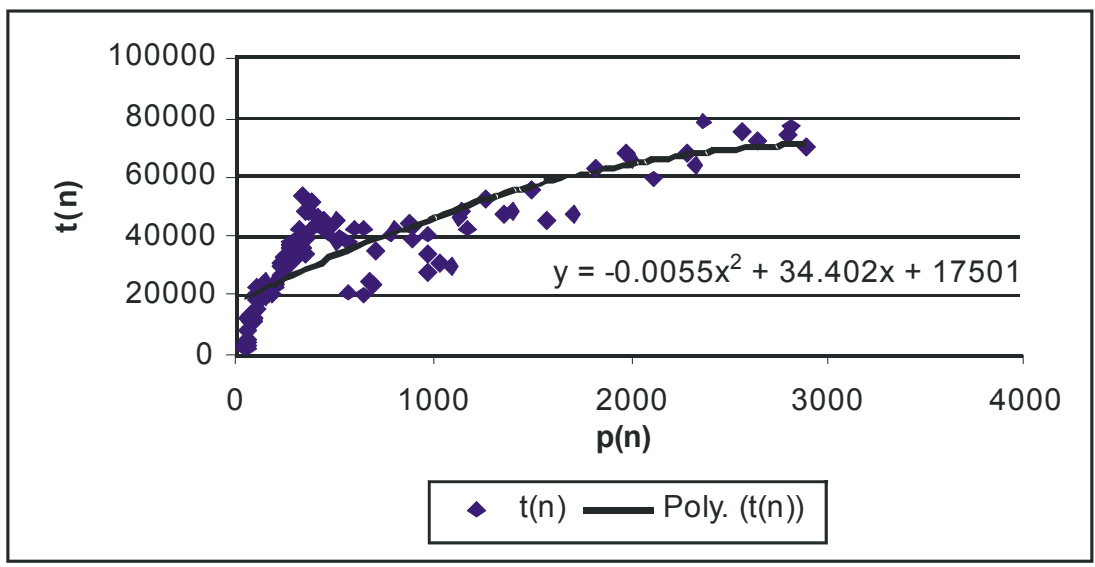

Finally, grouping the two equations we get:

$$
\begin{gathered}
p(n)=1+t(n-1)+a^{*} p(n-1)^{\wedge} 2 \\
t(n)=b^{*} p(n-1)
\end{gathered}
$$

By contrast to the behavior described by the quadratic equation with one parameter, the above system is called a Henon attractor and has a behavior presented in the figure below. 


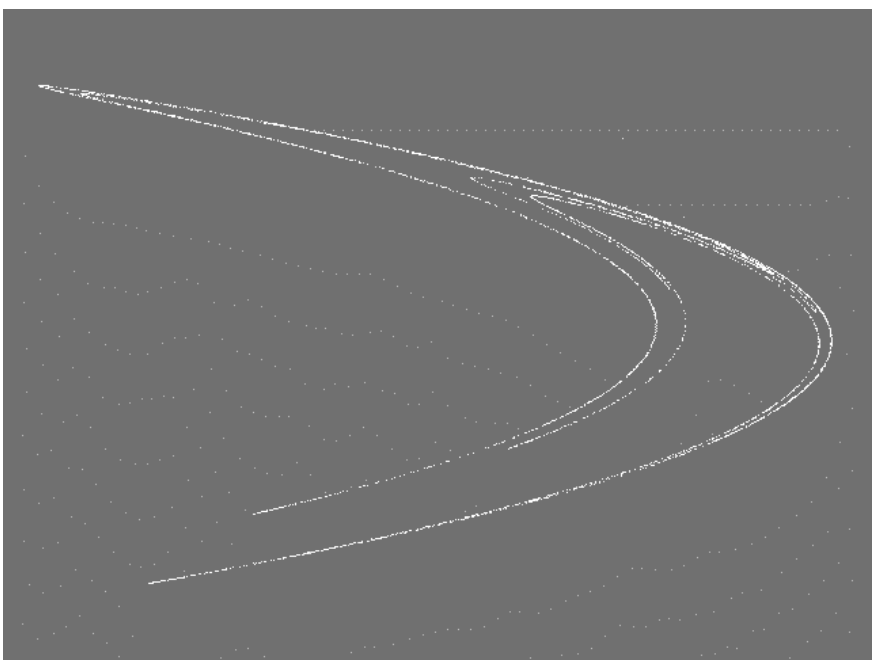

This type of basins of behavior has drawn the name of 'strange attractors' to the multitude of such complex mathematical entities (later on discovered).

The interesting thing about the occurrence of this behavior in the economies that along with the industrial production are allocating resources to enhance the production of technologies is the fact that one may describe various patterns of behavior that are not allowed by the usually used linear approach.

The behavior inside the basin of the attractor's dynamic shows some interesting features such as the fact that too little effort in technological knowledge production is associated to low industrial production, while too much technological production is also reaching an area of low industrial production.

This effect clearly shows that too little allocation of resources to research, that does not allow a large industrial production for lack of knowledge, is equivalent to a disproportionate allocation of resources to research that hampers the industrial production because of lack of resources for production. A badly managed economy may fall into the trap of producing large oscillations in allocating its resources, thus creating dynamic regimes whose consequences are hard to absorb and thus leading to an erratic evolution.

By contrast, there is an area of large industrial production that corresponds to a given rate of technologies production which, in some way, optimizes development. We have witnessed in the last decades several cases of economies, e.g. Ireland and Finland, in Europe, the Asian tigers and China, in Asia, that had had a moment in their history when research (both means and intelligence) was a main priority. Luckily, the globalization acted as a stabilizer that kept them close to the optimum of 'As High As Reasonably Acceptable' technologies production. By contrast to ALARA (as low as reasonably acceptable) principle, that applies in risk analysis, here we may introduce 


\section{Non-Linear Effects in Knowledge Production}

the AHARA principle resulting from the nonlinear interplay of technologies production and industrial production.

Although remaining parabolic in essence, the real behavior of the system may show different patterns for various coefficients determined from the experimental data. Data from the reference above may show a behavior described in the figure below.

\section{Production of technological knowledge and industrial production USA (1860-1976) 'Henon' strange attractor}

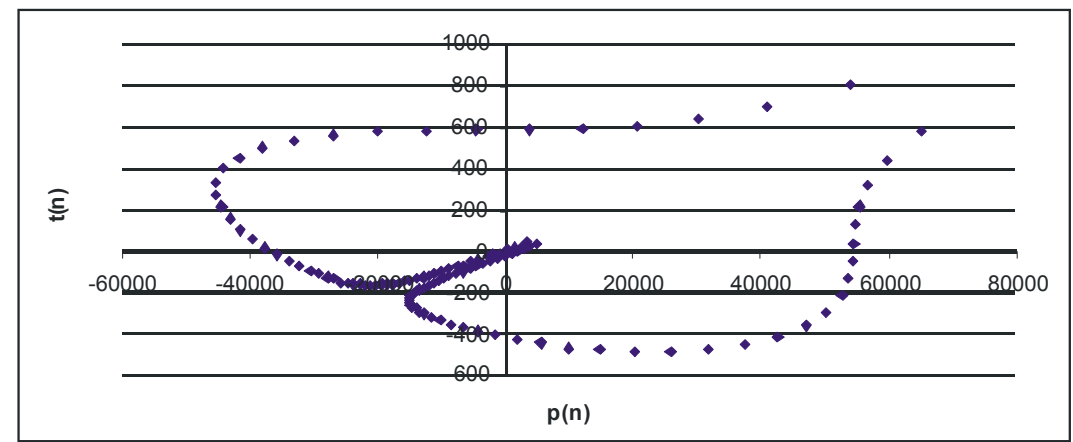

One may see that a strange attractor behavior is seen in this representation too having, in excess of the features explained above, the limitation of a certain value of industrial production over which the production of technological knowledge is increasing rapidly, still remaining within the attractor basin.

\section{Measuring technological information and entropy}

The comments on resource allocation made above are leading in a wider context to the problem of creation of technological information and further on to entropy, as a measure of economic transitions, taken in the sense by Georgescu-Roegen.

Since, in the case described above, the technological information and GDP productions' correlation, we have encountered the possibility for the occurrence of a chaotic regime with several trajectories of evolution confined to a 'strange attractor' (of Henon type), it is useful to analyze the specific parameters for such nonlinear dynamic.

The approach taken here will involve escort distributions and Renyi information measures but we will arrive there by first introducing a measure of information gain that is more appropriate, in our view, for economic processes that are strongly anchored in the experimental reality.

There is in the economic processes, like the one described above, an underlying logic that is not always a bivalent one. By recreating the conditions for technological knowledge generation, information is gained on the state of truth or falseness of the technological sequences under research and implementation. Let's consider that there are not only two states, i.e. true (functions, generates more GDP, is more 
efficient ...) and false (does not work, does not generate more GDP, is less efficient ...) of the technological sequence of events under consideration; but, any number of possible values in between true and false. Each of the intermediate values can be expressed as a combined measure of the measure of true and the one of the false technological sequences.

\section{Knowledge vector for technology sequence}

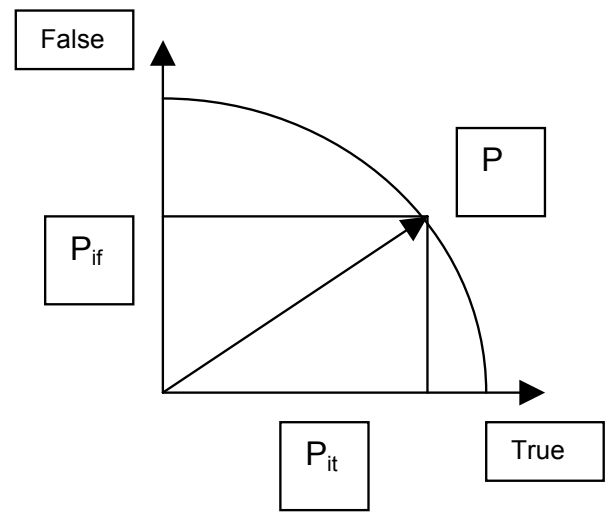

From the point of view of research and implementation we may define the specific measures of the two states as the probabilities that technological sequences will be true (in the sense above) defining, for true, the normalized probabilities for the technological sequence $i$ as $P_{i t}=\left(p_{i}\right)^{\beta} / \Sigma\left(p_{j}\right)^{\beta}$, where $p_{i}$ are not zero. Here the distributions $p$ are given by the observed relative frequencies of the technological experimental tests. In the same manner we define the measure of false $P_{\text {if }}$ for the sequence where instead of $p$ we take 1-p. The two measures are actually resulting from the research and development activity that separates the working technological events from the ones that do not work in the process to create useful technologies. Actually the research activity is creating conditions for testing the technological sequences and is gaining technological information from the outcome of the tests. The gain of information will be described below.

We may define a vector of the state of knowledge for each experimental stage in technological development and associate with it a measure that results from typical vector calculus as: $\mathrm{P}^{2}=\mathrm{P}_{\mathrm{it}}{ }^{2}+\mathrm{P}_{\text {if }}{ }^{2}$. Further on we consider that research and development is repeating the conditions for testing technologies and this is adding to the two dimensions (true and false) a third one that marks the passage of time; we are associating with this dimension a measure of time that results from the frequency of repeating the tests, noted $\mathrm{iP}_{0}$ (with $\mathrm{i}=$ sqrt(-1) marking a rotation by 90 degrees in the complex space). Since, after each development test, the vector of knowledge changes by gaining technological information, we may describe the change in information by 


\section{Non-Linear Effects in Knowledge Production}

the change of the knowledge vector in a space that has been defined as a Minkowsky space in physics. As shown in (Purica 1990) the gain in information is described by a Lorentz transformation. The resulting vector has a magnitude $P^{2}=P_{i t}{ }^{2}+P_{i f}{ }^{2}-P_{0}{ }^{2}$. Those familiar with relativity theory will recognize the specific three-dimensional cones that separate the space in three regions. These have a specific meaning in our interpretation: (i) inside the cone we have technological information that is generated from tests and still keeps a certain incertitude described by $\mathrm{P}_{0}{ }^{2}>\mathrm{P}_{\text {it }}{ }^{2}+\mathrm{P}_{\text {if }}{ }^{2}$. On the cone the technological information is coherent and leads to implementable technological sequences, while outside the cone $\mathrm{P}_{0}{ }^{2}<\mathrm{P}_{\mathrm{it}}{ }^{2}+\mathrm{P}_{\mathrm{if}}{ }^{2}$ (this would be a situation when the frequency of technological sequence's tests is smaller than the number of tests on technological knowledge which, situation is unreal).

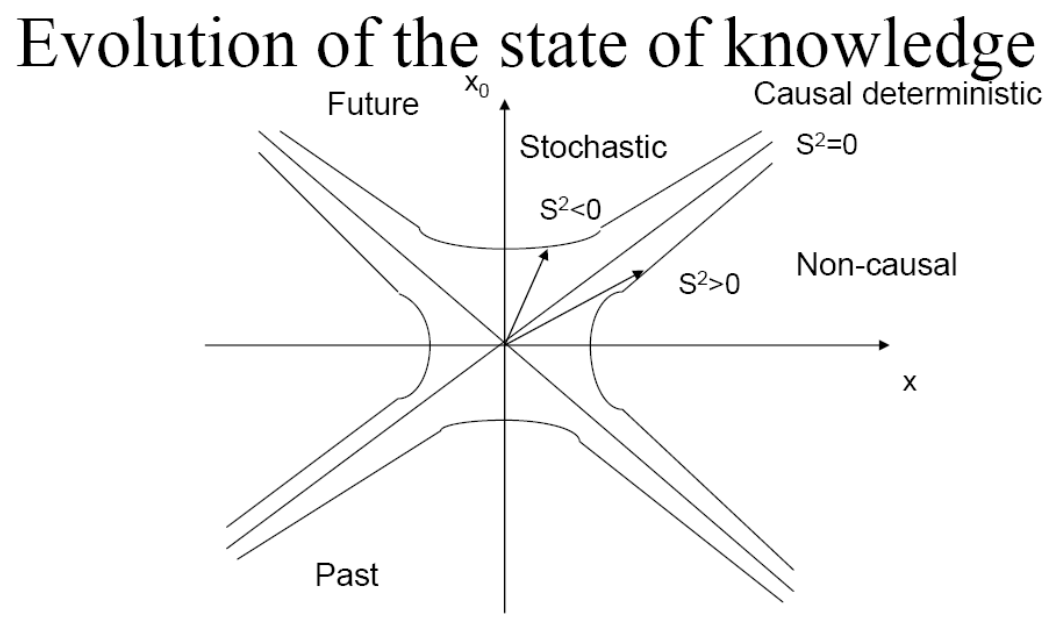

The pass from a state of knowledge to another is described by a Lorentz transform in the Minkovski space. The evolution of the state of knowledge is associated with a 'trajectory of knowledge'.

Coming back to the definition of the probabilities $\mathrm{P}$ we may take (Beck and Schlogl 1993) $1 / \beta$ as representing the equivalent of the temperature in the thermodynamic space. Thus the escort distribution considered above may be represented as dependent on a function that varies as a power of $\beta$. Moreover, for sequences of conditional technological events it may be factorized into two or more conditional probabilities leading to the equivalent of grand canonical ensemble with chemical potentials.

Measuring information of the process may be done (Beck and Schlogl 1993) by using the Renyi information of order $\beta$ of the original distribution given as: $I_{\beta}(p)=1 /(\beta-$ 1). $\ln \left(\Sigma\left(p_{\mathrm{j}}\right)^{\beta}\right)$. As a property of this measure, for $\beta=1$ the expression reduces to the one of Shannon information for the distribution $p$. The value of $\beta$ is associated with the inverse of temperature and serves to scan various behavior patterns of $p$ and its partition function that may determine a Helmholz free energy of the escort distribution as $F(\beta)=-1 / \beta \cdot \ln \left(\Sigma\left(p_{j}\right)^{\beta}\right)$. 
Institute of Economic Forecasting

\section{Conclusions}

Nowadays, the production of technological knowledge, through integrated research in the economic activities, is a must for the countries that strive for a coherent and consistent development, aimed at bringing them in the forefront of civilization.

In the interplay between the 'usual' industrial production and the production of technological knowledge, the rule is non-linear behavior and not the linear one. The simple thinking that more and more resources allocated to technological research would result in more industrial production may lead to the surprise of jeopardizing development.

By introducing a measure of the generation of technological knowledge through research and development we showed that gaining knowledge is associated with a Lorentz transformation in a Minkowski space of the frequencies of the tested sequences of technological events in time. The information measure for the associated escort distributions to these technological sequences is the Renyi one that suits itself better for the nonlinear dynamics of the process. To better specify the difference between information measure for the escort distributions and the knowledge gain one must stress that knowledge is referring to the experimental process that describes the interaction of the researching structures of the society with the technological sequences with the aim to produce implementable technologies; while the information measure is just describing each technology sequence in itself, making it comparable among them.

Obviously technological jump (on the logistic curve) may boost industrial production but, to enter the logistic it needs accumulated research. The decision to reallocate resources to a new technology is in itself a non-linear discontinuity in evolution. The 'Aha!' moment of creation is acting as a butterfly effect that generates a technological typhoon in the industry, but, it may also bring along costs that are impinging on the industrial production and are affecting the good present for a better future.

We are playing between ALARA in relation to accepting the risks and AHARA in relation to accepting costs of development. But, in any case, knowledge remains the basic element for setting both the low and the high limits of our evolution.

\section{References}

Baumol, W.J., "Unpredictability, Pseudorandomness and Military Civilian Budget Interactions", Revista Internationale di Science Economiche et Commerciali, April 1986, XXXIII:4, 297 -318.

Baumol, W. J. Benhabib, "Chaos: Significance, Mechanism, and Economic Applications", Economic Prospectives - A Journal of the American Economic Association, vol. 3, No I, Winter 1989.

Baumol, W. J. Wolff, E. N., "Feedback from Productivity Growth to R\&D", Scandinavian Journal of Economics, 1983, 85:2, 147-57. 
Non-Linear Effects in Knowledge Production

Grandmond Jean Michel, "Periodic and Aperiodic Behaviour in Discrete Onedimensional Dynamical Systems", in Hildenbrand, W and A Mass Coliel, eds., Contributions to Mathematical Economics, New York North Holland, 1986.

Purica, I. I., "Creativity, Intelligence and Synergetic Processes in the Development of Science", Scientometrics, Vol.13, Nos 1-2 (1988) p.11-24.

Purica, I. I., "Creativity and the socio cultural niche", Scientometrics, Vol.15, Nos 3-4 (1989) p.181-187.

Haustein, H-D, Neurath, E., "Long Waves in World Industrial Production, Energy Consumption, Innovations, Inventions and patents and Their Identification by Spectral Analysis", Technological forecasting and social change, 22, 53-89, 1982.

Purica, I. I., Legile gândirii modale, Editura Academiei, Bucureşti, 1990.

Beck, C., Schlogl, F., Thermodynamics of chaotic systems, Cambridge University Press, Cambridge, 1993. 